\title{
Lateralisation of speech dominance by spectral analysis of evoked potentials ${ }^{1}$
}

\author{
A. E. DAVIS ${ }^{2}$ AND J. A. WADA \\ From the Division of Neurological Sciences, Faculty of Medicine, University of British Columbia, \\ Vancouver, Canada
}

SUMMARY A computerised evoked potential test for the lateralisation of speech dominance was compared with the intracarotid sodium amylobarbitone test in 22 epileptic patients. Based on spectral analysis, the computer test correctly predicted the speech dominance of 15 of 16 left speech dominant and five of six right speech dominant patients. This non-invasive test is simple, quick, and innocuous and can be easily implemented on a small laboratory computer.

It has been known since at least the time of Dax and Broca that lesions of the left temporal areas result in disturbances of many aspects of language (Geschwind, 1970; Milner, 1974). Present evidence, however, shows clearly that the lateralisation of cerebral representation of speech is only one aspect of a complementary specialisation of the dominant and non-dominant hemispheres for the processing of verbal and visuospatial information respectively (Milner, 1974; Sperry, 1974; Teuber, 1974).

We have investigated these asymmetries in normal right- and left-handed adults (Davis and Wada, 1974, 1976), left and right speech dominant epileptics (Davis and Wada, 1974), and infants (Davis and Wada, 1976). From this research, a computer test for the determination of the lateralisation of speech dominance has been developed. The approach is based on the spectral analysis of visual and auditory evoked potentials (VEPs and AEPs). It involves measuring the coherence or similarity of form between occipital and temporal evoked potentials produced in response to flash and click stimuli. The major observation of our earlier work on a small number of patients is that click stimuli generate more coherent responses within the speech dominant hemisphere, while flash stimuli generate more coherent responses within the non-dominant hemisphere. The prediction of speech dominance thus

\footnotetext{
1 This work was supported by a Canadian Medical Research Council grant to J. A. Wada.

${ }^{2}$ Address for correspondence: Dr Alan E. Davis, Division of Neurological Sciences, University of B.C., Vancouver, B.C., Canada. (Dr Davis is a Canadian M.R.C. Postdoctoral Fellow.)

Accepted 12 July 1976
}

involves a consideration of the complementary specialisation of both hemispheres in response to visual and auditory stimuli.

\section{Methods}

The patients were 16 left speech dominant (LSD), 11 male and five female, and six right speech dominant (RSD), three male and three female, aged from 12 to 43 years. They were being evaluated for possible surgical treatment of medically intractable seizure disorders. A majority (19) had either left or right temporal lobe foci and three had foci in the frontal lobe. At the time of testing they were on continued medications including phenytoin, methoin, phenobarbitone, and carbamazepine.

Carotid amylobarbitone (amytal) speech lateralisation (Wada, 1949; Wada and Rasmussen, 1960) was carried out with femoral catheterisation under local anaesthesia, with 75-100 mg sodium amylobarbitone in 3-4 ml solution injected into the internal carotid artery. The test was performed bilaterally at least two days apart. Concurrently, a hand injection carotid arteriogram with a comparable amount of dye and simultaneous EEG monitoring were always done to aid interpretation of the clinical results.

Handedness was determined from a questionnaire consisting of 12 criteria (Annett, 1967). These included the eye, hand, or foot used to do the following tasks: writing, throwing a ball, striking a match, holding scissors, holding a thread in threading a needle, dealing cards, holding a hammer, holding a toothbrush, unscrewing a jar-lid, holding a tennis racket, looking through a microscope, and kicking 
a ball. Patients were classified as 'pure' right- or left-handed if they performed at least 10 of the 12 tasks with one side of their body only, and were classified as 'mixed' if they did not.

Visual and auditory evoked potentials (VEPs and AEPs) generated in response to flash and click stimuli were recorded from the left and right occipital and temporal areas ( $\mathrm{O} 1, \mathrm{O} 2, \mathrm{C} 5$, and $\mathrm{C} 6)$ electrodes on the international 10-20 system). Linked right and left earlobes were chosen as the reference because they do not show any consistently time-locked evoked activity with respect to any number of other sites (Goff et al. 1969; Regan, 1972).

The responses to 160 flash and 160 click stimuli, randomly presented at least two seconds apart, were averaged into a single VEP and single AEP from each of the four recording areas. These EPs were then spectrum analysed to give the coherence between the occipital and temporal responses in each hemisphere. More detail is included in the Appendix for those interested in implementation. The procedures were standard spectral analysis techniques available on most small laboratory computers such as PDP 8.

The Fisher exact probability non-parametric test for paired comparisons (Conover, 1971) was used for all statistical analyses. This test made no assumptions about normal distributions. It was used to determine if LSD and RSD patients had significantly different coherence asymmetries between the left and right hemispheres.

\section{Results}

The results of the comparisons between speech dominance, coherence asymmetries and handedness are shown in the Table. Both flash and click stimuli produced significantly different coherence asymmetries between the LSD and RSD patients ( $P$ less

Table 1. Number of patients with maximumoccipital-to temporal coherence in left or right hemisphere for click stimuli, and 2. for flash stimuli. 3. Number of patients with right or left-and mixed-handedness. 4. Consensual prediction of speech dominance from flash and click coherence asymmetries and handedness

\begin{tabular}{|c|c|c|c|c|c|}
\hline Asymmetry & $\begin{array}{l}L S D \\
\left(10^{\circ} p\right. \\
\text { Left }\end{array}$ & $\begin{array}{r}\text { atients) } \\
\text { Right }\end{array}$ & $\begin{array}{l}R S D \\
(6 \mathrm{pa} \\
\text { Left }\end{array}$ & $\begin{array}{l}\text { ients) } \\
\text { Right }\end{array}$ & $P_{L S D}=$ RSD \\
\hline $\begin{array}{l}\text { 1. Maximum click } \\
\text { 2. Maximum flash } \\
\text { 3. Handedness } \\
\text { 4. Consensual } \\
\text { dominance }\end{array}$ & $\begin{array}{r}13 \\
3 \\
4 \\
15\end{array}$ & $\begin{array}{r}3 \\
13 \\
12 \\
1\end{array}$ & $\begin{array}{l}1 \\
4 \\
4 \\
1\end{array}$ & $\begin{array}{l}5 \\
2 \\
2 \\
5\end{array}$ & $\begin{array}{l}0.0105 \mathrm{P}<0.025 \\
0.0493 \mathrm{P}<0.050 \\
0.0854 \mathrm{NS} \\
0.0013 \mathrm{P}<0.005\end{array}$ \\
\hline
\end{tabular}

(LSD, RSD = left, right speech dominants). $P_{L S D=R S D}$ is the probability that the asymmetry is the same for LSD and RSD patients. than 0.05). For click stimuli, the dominant hemisphere had more coherent responses in 13 of 16 LSD patients and five of six RSD patients $(P=0.0105)$. Conversely, for flash stimuli, 13 of 16 LSD patients and four of six RSD patients had larger coherences in the non-dominant hemisphere $(\mathrm{P}=0.0493)$. Handedness was not significantly related to speech dominance if left- and mixed-handed patients were grouped together $(P=0.0854)$. Most right-handed patients (12 of 14) were LSD, while left- and mixedhanded patients were evenly divided between LSD and RSD groups (four of eight LSD and four of eight RSD).

The Table also shows that the most reliable prediction of speech dominance was a consensual measure of flash and click coherence asymmetries and handedness. In this measure, a patient was classified as LSD if he had two of the three following: maximum click coherences in the left hemisphere, maximum flash coherences in the right hemisphere, and right handedness. Conversely, he was classified as RSD if he had two of the three following: maximum click coherence in the right hemisphere, maximum flash coherence in the left, and left- or mixed-handedness. With this consensual measure, 15 of 16 LSD and five of six RSD patients were correctly classified $(\mathrm{P}=\mathbf{0 . 0 0 1 3})$.

\section{Discussion}

The above results show that click and flash stimuli produce significantly different hemispheric asymmetries of coherence. These are significantly related to speech dominance, as they are reversed between LSD and RSD groups. Intuitively, the results mean that the form of occipital and temporal responses are more similar within the dominant hemisphere for click stimuli and within the non-dominant hemisphere for flash stimuli. The functional significarce of these asymmetries is discussed elsewhere (Davis and Wada, 1976).

As expected, handedness was not significantly related to speech dominance (Heilman et al. 1973, 1974). However, in this group, left- or mixedhanded patients were more likely to be RSD than were right-handed patients. This observation has also been made in studies of the effects of lesions (Hécaen and Ajuriaguerra, 1964), electrostimulation of the exposed cortex (Penfield and Roberts, 1959), and the carotid amytal test (Branch et al. 1964; Milner et al., 1966).

The consensual prediction of speech dominance by click and flash coherence asymmetries and handedness is very accurate. Only one LSD and one RSD patient out of 22 were misclassified by this procedure, an accuracy of greater than $90 \%$. The 
amytal test itself is about $95 \%$ accurate as determined by speech disturbances resulting from excision of the amytal-predicted, non-dominant temporal lobe (Branch et al., 1964).

Both the coherence and consensual measures were much superior to handedness as predictors of speech dominance. Handedness was not significantly related to speech dominance, and was only of use in correctly classifying 12 of the 14 right-handed patients as LSD. The remaining eight non-righthanders could not have been predicted by their handedness, since four were LSD and four were RSD. The handedness prediction would thus have been two incorrect classifications as LSD and eight unknown in a total of 22 patients, an uncertainty or inaccuracy of nearly $50 \%$. In contrast, only one LSD and one RSD patient were misclassified by the consensual test-an accuracy of greater than $90 \%$.

The amytal test has been, and will continue to be, of primary importance in the diagnostic evaluation of a patient's suitability for the surgical treatment of epilepsy. The computer test is now being used as a supportive measure in these investigations. In addition, it can be used in circumstances where the amytal test is not applicable. These might include clinical investigations of uncooperative or uncommunicative patients, those with communication disorders associated with dyslexia or autism, and longitudinal studies of normal and abnormal speech development.

In conclusion, the combination of evoked potential and behavioural tests allows a completely noninvasive determination of speech dominance. The accuracy of the prediction is much superior to one based on handedness and rivals that of the amytal test. The technique makes use of the concept that speech dominance is only one aspect of a complementary hemispheric specialisation of verbal, visuospatial, and motor functions. We are currently developing these tests with complex stimuli containing verbal and pictorial information. We fully expect that these will lead to further clinical applications of the fundamentally important concept of hemispheric asymmetry.

\section{References}

Annett, M. (1967). The binomial distribution of right, mixed and left handedness. Quarterly Journal of Experimental Psychology, 19, 327-333.

Branch, C., Milner, B., and Rasmussen, T. (1964). Intracarotid sodium amytal for the lateralization of cerebral speech dominance. Journal of Neurosurgery, 21, 399-405.

Conover, W. J. (1971). Practical Non-Parametric Statistics. Wiley: New York.

Davis, A. E., and Wada, J. A. (1974). Hemispheric asymmetry: frequency analysis of flash and click evoked responses to non-verbal stimuli. Electroencephalography and Clinical Neurophysiology, 37, 1-9.

Davis, A. E., and Wada, J. A. (1976a). Spectral analysis of evoked potential asymmetries related to speech dominance. In Cerebral Evoked Potentials in Man. Edited by J. Desmedt. Oxford University Press: London.

Davis, A. E., and Wada, J. A. (1976b). Hemispheric asymmetry in neonates: demonstration by spectral analysis. Brain and Language, accepted.

Dixon, W. J. (1973). BMD: Biomedical Computer Programs. University of California Press: Berkeley.

Geschwind, N. (1970). The organization of language and the brain. Science, 170, 940-944.

Goff, W. R., Matsumiya, Y., Allison, T., and Goff, G. D. (1969). Cross-modality comparisons of averaged evoked potentials. Average Evoked Potentials, pp. 95-118. Edited by E. Donchin and D. B. Lindsley. NASA SP-191: Washington.

Hécaen, H. and Ajuriaguerra, J. (1964). Left-handedness, Manual Superiority and Cerebral Dominance. Grune and Stratton: New York.

Heilman, K. M., Coyle, J. M., Gonyea, E. F., and Geschwind, N. (1973). Apraxia and agraphia in a left-hander. Brain, 96, 21-28.

Heilman, K. M., Gonyea, E. F., and Geschwind, N. (1974). Apraxia and agraphia in a right-hander. Cortex, 10, 284-288.

Jenkins, G. M. and Watts, D. G. (1968). Spectral Analysis and its Applications. Holden-Day: San Francisco.

Koopmans, L. H. (1974). The Spectral Analysis of Time Series. Academic: New York.

Milner, B. (1974). Hemispheric specialization: scope and limits. In The Neurosciences: 3rd Study Program, pp. 75-89. Edited by F. O. Schmitt and F. G. Worden. MIT Press: Cambridge, Mass.

Milner, B., Branch, C., and Rasmussen, T. (1966). Evidence for bilateral speech representation in some non-right handers. Transactions of the American Neurological Association, 91, 306-308.

Penfield, W., and Roberts, L. (1959). Speech and Brain Mechanisms. Princeton University Press, Princeton, NJ.

Regan, D. (1972). Evoked Potentials in Psychology, Sensory Physiology and Clinical Medicine. Chapman and Hall: London.

Sperry, R. W. (1974). Lateral specialization in the surgically separated hemispheres. In The Neurosciences: 3rd Study Program, pp. 5-19. Edited by F. O. Schmitt and F. G. Worden. MIT Press: Cambridge, Massachusetts.

Teuber, H. L. (1974). Why two brains? In The Neurosciences: 3rd Study Program, pp. 71-74. Edited by F. O. Schmitt and F. G. Worden. MIT Press: Cambridge, Massachusetts.

Wada, J. A. (1949). A new method for the determination of the side of cerebral speech dominance. A preliminary report on the intracarotid injection of sodium amytal in man. Medicine and Biology (Japanese), 14, 221-222.

Wada, J. A., and Rasmussen (1960). Intracarotid injection of sodium amytal for the lateralization of cerebral speech dominance. Journal of Neurosurgery, 17, 262-282. 


\section{Appendix}

This section is not necessary for an understanding of the technique, but is required information for implementation.

Each 500 milliseconds, 50 point sample, average evoked potential (EP) of 160 stimuli was first prefiltered with a three point, 1-2-1 weighted moving average filter. This removed frequencies above about $30 \mathrm{~Hz}$. For each subject and stimulus modality, the four averaged EPs from the four recording areas were analysed with the UCLA Biomedical computer programme, BMD 03T-Time series spectrum estimation (Dixon, 1973). Non-stationary linear trend was first removed from each series, and then each series was decomposed into frequency components by means of a fast-Fourier transform. Estimates of the coherence and cross-spectrum were obtained by summing products of the transformed series.

The spectral analysis produced coherences of 6 $\mathrm{Hz}$ resolution between the occipital and temporal areas of each hemisphere. The coherence at the frequency of greatest cross-spectral or shared activity was used to compare the left and right hemispheric responses. This frequency range was $3-9 \mathrm{~Hz}$ in 18 patients, and $0-3 \mathrm{~Hz}$ in four patients.
Spectral analysis is a primarily descriptive technique which is useful in exploring the basic characteristics of mechanisms which generate oscillating waveforms (Jenkins and Watts, 1968; Koopmans, 1974). Its two major assumptions are, first, that the statistical properties of the generating mechanism are stationary and do not change over the time interval of interest, and second that individual points in the waveform follow a normal sampling distribution. The violation of these assumptions, however, does not present serious problems and only slightly reduces the reliability of the estimates (Jenkins and Watts, 1968; Koopmans, 1974).

In practice, 'real' data never completely satisfy the assumptions of spectral analysis. The technique thus provides guidelines and descriptions of the process rather than exact incontrovertible answers. In the final analysis, the 'proof' of a good design is that the results of the analysis make good physical sense (Koopmans, 1974). This is the case in the present report. A consistent relationship between coherence and speech dominance has been demonstrated, and this is meaningful in terms of the concepts of complementary specialisation of hemispheric functions. 